\title{
LO QUE LOS GESTOS DICEN Y LAS PALABRAS CALLAN: UNA ENTREVISTA CON CLINTON
}

\author{
Gloria Álvarez-Benito \& Isabel M. Íñıgo-Mora \\ Universidad de Sevilla
}

\begin{abstract}
Resumen: Este artículo se centra en el estudio de las entrevistas televisadas, un campo sobre el que existe una amplia literatura (Bull y Elliot 1998; Carter y Mccarthy 2002; Clayman 1988, 1991, 1992, 1993; Greatbatch 1986, 1988, 1992; Harris 1986, 1991; y Heritage y Greatbatch 1991). Nuestro objetivo consiste en analizar el uso de los mecanismos verbales y no verbales como estrategias comunicativas en las entrevistas políticas, y más concretamente en una entrevista televisada con el ex-Presidente de los Estados Unidos, Bill Clinton. Pretendemos descubrir cómo los politicos intentan sacar ventaja de los mecanismos verbales y no verbales en las entrevistas televisadas.
\end{abstract}

Palabras clave: verbal, no verbal, estrategias, gestos, paralenguaje.

Abstract: This paper focuses on the study of televised interviews, a field of which there is a vast amount of literature (Bull and Elliot 1998; Carter and Mccarthy 2002; Clayman 1988, 1991, 1992, 1993; Greatbatch 1986, 1988, 1992; Harris 1986, 1991; and Heritage and Greatbatch 1991). Our objective is to analyse the use of verbal and nonverbal elements as communicative strategies in political interviews and more specifically in a televised interview with the ex-President of the United States, Bill Clinton. We will attempt to discover the way in which politicians try to take advantage of verbal and nonverbal signals in televised interviews.

Keywords: verbal, nonverbal, strategies, gestures, paralanguage.

"Los políticos mienten cuando afirman, mienten cuando niegan, y sobre todo mienten cuando callan” (Miguel de Unamuno)

\section{INTRODUCCIÓN}

Los políticos de todo el mundo hacen uso de los medios de comunicación, principalmente porque son conocedores del enorme poder que representan y también de que es el mejor medio para llegar a un gran número de personas. Este tipo de comunicación difiere estructuralmente de la comunicación cara a 
cara, pues suele conllevar una disyunción de lugar y de tiempo entre el comunicador y la audiencia (Alan, 1991). Según Tracey (1977:6) la comunicación política consiste en "... Channels for the transmission of messages from political actors to their political audience." Sin embargo, es importante señalar que la comunicación de masas también entraña un riesgo considerable, ya que si el político en cuestión no hiciera una buena actuación, entonces podría poner su carrera política en serio peligro.

Las entrevistas televisadas constituyen un área de la comunicación de masas de gran importancia, prueba de ello es el gran interés que suscitan y la extensa literatura que hay publicada al respecto (Bull y Elliot 1998; Carter y Mccarthy 2002; Clayman 1988, 1991, 1992, 1993; Greatbatch 1986, 1988, 1992; Harris 1986, 1991; y Heritage y Greatbatch 1991). En este artículo tenemos como objetivo principal analizar los recursos verbales y no verbales utilizados por el político en las entrevistas. Nuestro interés se centra, por lo tanto, no sólo en lo que se dice, sino también en cómo se dice. Es decir, nuestro objeto de análisis será la palabra (estrategia verbal), pero también lo serán el paralenguaje, los gestos, la postura, el contacto visual, etc., que (co)ocurren con la palabra. Tal y como argumenta Sornig (1989:109) "Persuasive use of language does not so much appeal to reason but to the recipient's expectations and emotions." $Y$ no podemos obviar que el político puede influir en las emociones y expectativas del receptor tanto por medio de estrategias verbales como no verbales.

Las entrevistas televisadas realizadas a miembros de instituciones políticas y representantes del gobierno difieren de las conversaciones comunes en el hecho de que hay una preasignación de los turnos de palabra (Atkinson y Drew 1979; Greatbatch 1988). La entrevista debe estar estructurada en secuencias de preguntas y respuestas. Los entrevistadores (IRs) deben limitarse a formular preguntas y los entrevistados (IEs) deben abstenerse de hacer preguntas o hacer comentarios no requeridos sobre cuestiones previas y por lo tanto deben limitarse a responder la preguntas hechas por el IR (Heritage y Greatbatch 1991). Según Heritage (2003), cuando un IR formula una pregunta se enfrenta a dos normas periodísticas que se encuentran en clara competencia: mostrar imparcialidad y crear tensión. A este respecto, Heritage (2003:57) señala:

(...) IRs are expected to be impartial, objective, unbiased, and disinterested in their questioning of public figures. They are expected to have respect for the facts and the perspectives that IEs communicate, and to work to being these into public domain.

Por una parte, los entrevistadores no pueden ser los portavoces de los entrevistados, sino que más bien los deben desafiar; pero por otra parte, como ya han comentado Heritage y Roth (1995:1-2), en la mayoría de las sociedades occidentales no se espera que los IRs debatan o argumenten con los IEs o critiquen 
sus puntos de vista, pero tampoco que los IRs defiendan, apoyen o muestren su acuerdo con los puntos de vista de los IEs.

Sin embargo, un buen IR debe conseguir la máxima información posible del IE y por este motivo puede que tenga que hacer preguntas que resulten incómodas para el IE. Además, estas preguntas incómodas deberían crear la máxima conmoción o agitación posible. Sólo los IRs que tienen la habilidad de hacer este tipo de preguntas son famosos o conocidos. Por otro lado, un IR es un ser humano que tiene un ideología y una postura política determinadas y por ese motivo, puede verse tentado a hacer que el IE revele la información que al entrevistador le interesa. Los políticos, de todas maneras, no suelen responder a todas las preguntas que les hacen (Blum-Kulka 1983; Bull 1994; Bull y Mayer 1993; Clayman 1993 y 2001, y Harris 1991), al menos de manera verbal. Por ese motivo, se hace necesario consideren no sólo el comportamiento verbal del entrevistado, sino también el no verbal. Los elementos verbales y los no verbales juegan un papel igualmente importante en el discurso, existiendo entre ellos una relación de interdependencia. Las palabras co-ocurren con gestos y elementos paralingüísticos (cualidades vocales, inflexiones de la voz, etc.) para crear significado. Muchos investigadores han señalado el hecho de que para analizar el discurso hay que tener en cuenta tanto la comunicación verbal como la no verbal (McNeill 1985, Poyatos 2002, y Richmond, McCroskey y Payne 1991).

A pesar de que, tal y como hemos mencionado, los elementos verbales y los no verbales tienen una relación de interdependencia, sin embargo, los no verbales pueden, $\mathrm{y}$ de hecho suelen, tener más poder comunicativo que las propias palabras. Una de las razones para que esto sea así es que, habitualmente, prestamos más atención a cómo se dicen las cosas que a lo que se dice, en palabras de Hall (1973:2) "What people do is frequently more important than what they say". De hecho, cuando las palabras y los gestos emiten mensajes contradictorios, se tiende a dar más fiabilidad a los segundos. La apariencia, los gestos faciales, los movimientos de manos, las sonrisas, las posturas, la mirada, el contacto visual aportan, con o sin palabras, información al receptor. Los políticos son conscientes del poder que estos elementos comunicativos pueden tener sobre la audiencia, saben que su lenguaje corporal y sus cualidades vocales pueden ayudarles a proyectar una determinada imagen y por eso suelen hacer un gran esfuerzo para sacar el máximo provecho de ellos.

Si bien el uso de elementos no verbales es relevante en cualquier tipo de entorno comunicativo, es en el discurso televisado en el que estos mecanismos ocupan un lugar especialmente importante, quizá por el impacto visual que pudieran tener sobre la audiencia. Según Hellweg, Pfau y Brydon (1992:79) "Television devaluates content in political discourse, instead stressing candidate image, a by-product of the importance of the visual emphasis in television communication". De hecho, no es de extrañar que el mismo discurso pueda 
interpretarse de manera diferente dependiendo de si la audiencia lo ha visto por televisión o lo ha oído por la radio.

El presente estudio se va a centrar en el análisis discursivo de una entrevista de televisión realizada a Bill Clinton en el año 2004, cuando ya no era Presidente de los Estados Unidos. Una de las razones por la que hemos seleccionado esta entrevista es porque Clinton no sólo debe su fama al hecho de haber sido Presidente de los Estados Unidos durante dos mandatos (1993-2001), sino también por su relación extramarital con Monica Levinsky y el consiguiente proceso de acusación en 1998. Sabemos que el entrevistador no sólo haría preguntas relacionadas con asuntos políticos, sino que también indagaría sobre la vida privada de Clinton y su escándalo sexual, lo cual podría poner al entrevistado en una situación poco agradable. Clinton ha sido siempre un experto en el arte de evitar las preguntas incómodas y sería interesante analizar hasta qué punto es también un experto en enmascarar su comunicación no verbal cuando evita esas preguntas.

\section{METODOLOGÍA}

En esta sección pretendemos ofrecer una descripción detallada del material objeto de este estudio, sobre su transcripción, codificación y etiquetado, así como de los criterios seguidos en el análisis.

Material: el material a analizar es una entrevista de 45:18 minutos de duración del programa Panorama (A Panorama Special) emitido por la BBC1 el 22 de junio de 2004 a las 10:35pm y que tuvo lugar en un hotel de Nueva York. David Dimbleby entrevistó a Bill Clinton, el cual concedió esta entrevista a Panorama como parte de la campaña de publicidad de su autobiografía My Life.

Transcripción y Codificación: El punto de partida de esta sección fue una primera transcripción obtenida a través de la página web del canal de comunicación (http://news.bbc.co.uk/1/hi/programmes/panorama/3885521. stm). Esta transcripción inicial nos sirvió de guía preliminar para la posterior realización de una transcripción más exacta y detallada en la que se incluyó tanto información verbal como no verbal. Seguidamente se codificaron y anotaron los aspectos que se indican a continuación, y que se explicarán con más detalle en la sección denominada "Criterios para el análisis de datos", un poco más adelante en este mismo apartado sobre metodología:

1. Numeración y etiquetado de los turnos de palabra.

2. Clasificación y etiquetado de los turnos de palabra según su contenido verse sobre asuntos públicos o asuntos de la vida privada del entrevistado: "contenido público" y "contenido privado".

3. Identificación y cómputo de los elementos paralingüísticos que se utilizan en la entrevista. 
4. Identificación y cómputo de los gestos faciales adoptados por Clinton a lo largo de la entrevista.

5. Identificación y cómputo de los gestos de Clinton realizados con las manos, sus movimientos corporales y posturas.

6. Análisis del contacto visual entre el entrevistador y el entrevistado.

7. Identificación y cómputo de las estrategias verbales del discurso del entrevistado.

8. Sincronía de los elementos no verbales con otros mecanismos y estrategias verbales y no verbales.

9. Valoración del estado emocional de Clinton en los diferentes turnos de palabra.

Se hace necesario mencionar que, en lo que se refiere al etiquetado y codificación de estas nueve categorías, no hubo discrepancias entre las dos personas que llevamos a cabo esta labor de anotación. En consecuencia, los dos investigadores coincidimos en las anotaciones, incluso en aquellas categorías que podrían considerarse más subjetivas (como es el caso de la categoría número 9, 'valoración del estado emocional de Clinton').

Criterios de Análisis de Datos: El primer paso ha sido numerar todos los turnos de palabra, de manera que sea más fácil referirnos a ellos en la subsiguiente codificación, así como en los resultados y en la discusión. Se han utilizado las claves $Q_{2}$ para etiquetar los turnos que correspondían a preguntas (=Question) (es decir, los turnos del entrevistador Dimbleby), y A para las respuestas (=Answer) (es decir, los turnos de Clinton). Estas claves, Qo A, van seguidas de un número que indica su orden de aparición en la entrevista. La entrevista objeto de nuestro análisis consta de 122 turnos, etiquetados como Q1/A1 hasta Q61/A61. E1 segundo paso ha consistido en etiquetar los 122 turnos como "público" o "privado", dependiendo de si el contenido sobre el que trata dicho turno pertenece al ámbito público o privado de la vida de Clinton. Los turnos se han etiquetado del 1 al 6 según los siguientes criterios:

1. El contenido es privado, relacionado con asuntos de la vida familiar de Clinton.

2. El contenido del turno es también privado, pero en este caso se refiere al escándalo sexual del entrevistado con Monica Levinsky.

3. El contenido hace referencia a asuntos públicos, relacionados con la política internacional.

4. El contenido hace también referencia a asuntos públicos, pero en este caso se refieren a la política doméstica o nacional. 
5. El contenido se puede considerar tanto privado como público. Es decir, tiene que ver con decisiones políticas adoptadas por Clinton pero que se relacionan con su escándalo sexual.

6. El contenido es, de nuevo, privado y público a la vez, pero en este caso los asuntos políticos se relacionan con la vida familiar de Clinton.

También se ha utilizado la etiqueta (0) cuando el contenido se consideraba irrelevante y, por lo tanto, no tenía ningún tipo de relación ni con la vida pivada de Clinton ni con la pública (i.e. "Mr President thank you").

$\mathrm{E} 1$ tercer paso ha sido identificar los elementos paralingüísticos, que son mecanismos vocales no verbales, cualidades de la voz que afectan y tienen una gran incidencia en lo que se dice (ritmo, velocidad, entonación, tono, etc.).

El paso siguiente ha consistido en identificar los gestos faciales utilizados en los turnos de palabra, centrándonos en los más relevantes o sorprendentes y analizando su función comunicativa (discutiendo en algunas ocasiones sobre su naturaleza consciente o inconsciente).

En el paso posterior se han identificado y anotado los movimientos corporales y la postura, analizando también su función comunicativa.

A continuación, la codificación y etiquetado se ha centrado en el contacto visual entre los dos participantes en la interacción (Clinton y Dimbleby), aunque nuestro foco principal de análisis ha sido el entrevistado. Se han distinguido y anotado dos situaciones:

1. contacto visual, $\mathrm{y}$

2. ausencia de contacto visual.

El objetivo del paso 7 ha sido la identificación de las estrategias verbales utilizadas a lo largo de la entrevista: listas de tres, contrastes, Extreme Case Formulations (ECFs), Formulaciones Esquemáticas (Script Formulations), metonimias, etc. Todos estos recursos serán tratados en profundidad en la sección de discusión.

Seguidamente, se ha analizado la sincronización entre habla-gestos. Los elementos verbales y no verbales han sido codificados como:

1. Mecanismo simple:

1a) cuando una o más estrategias verbales se utilizan de manera aislada (ie. el mecanismo verbal no co-ocurre con un mecanismo no verbal).

1b) cuando uno o más elementos no verbales se utilizan de manera aislada (ie. el mecanismo no verbal utilizado no co-ocurre con una estrategia verbal).

2. Mecanismo Compuesto: cuando los mecanismos o estrategias verbales y no verbales co-ocurren en sincronía. 
Por último, se han puntuado los turnos de Clinton del 1 al 3, dependiendo del estado emocional: (1) relajado, (2) enfadado, y (3) muy enfadado o colérico (tensión extrema).

\section{RESULTADOS}

La Figura 1 que aparece a continuación muestra el uso que Bill Clinton hace de las estrategias verbales a lo largo de toda la entrevista, en los 61 turnos de palabra que tiene.

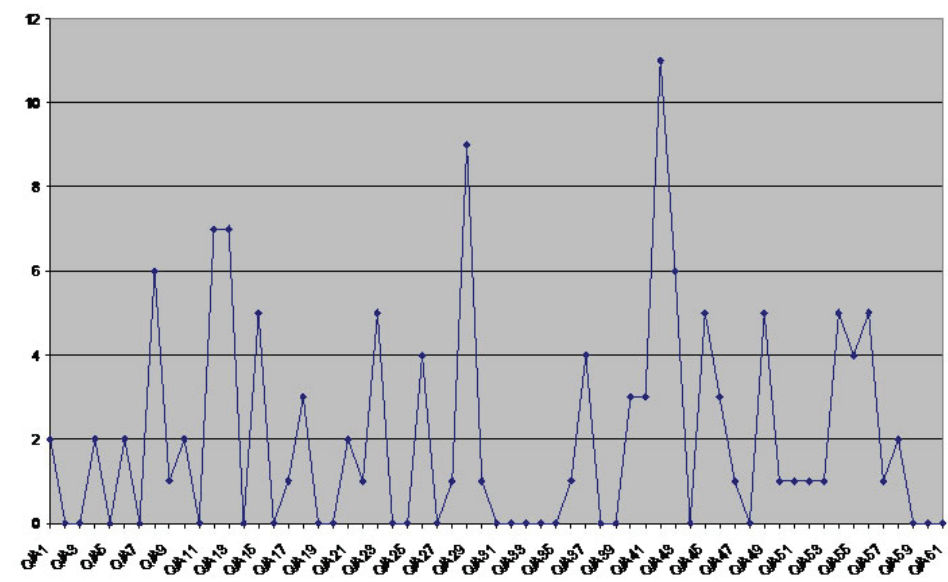

Figura 1. Estrategias verbales de Clinton

La Figura 2 ilustra el estado emocional de Clinton a lo largo de los diferentes turnos de palabra de la entrevista con Dimbleby.

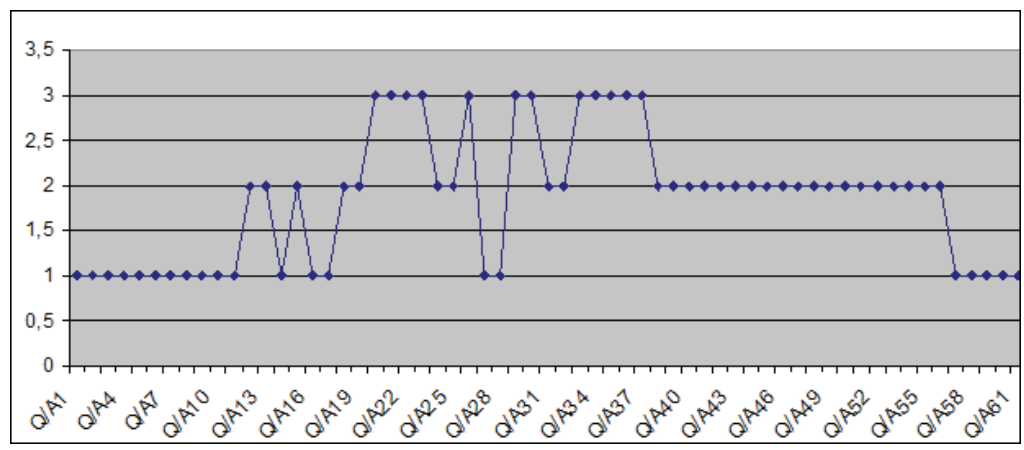

Figura 2. Estado emocional de Clinton 
En la Tabla 1 se muestran las correlaciones halladas entre las variables "verbal"y "no verbal", a fin de analizar y discutir posteriormente las posibles relaciones entre ambas".

\begin{tabular}{|l|l|r|r|}
\hline \multicolumn{4}{|c|}{$\begin{array}{c}\text { Tabla 1: Correlación de las variables } \\
\text { "verbal” "y “no verbal” }\end{array}$} \\
\hline & & no verbal & \multicolumn{1}{|c|}{ verbal } \\
\hline no verbal & Correlación de Pearson & 1 & $\mathbf{0 . 7 9 5}$ \\
\hline & N & 61 & 61 \\
\hline verbal & Correlación de Pearson & $\mathbf{0 . 7 9 5}$ & 1 \\
\hline & N & 61 & 61 \\
\hline
\end{tabular}

Como se ha podido apreciar en la Tabla 1, la correlación existente entre los elementos verbales y los no verbales es alta (0.795), los cual implica que las dos variables tienden a aumentar y disminuir a la vez. Estos datos se analizarán con más detalle en la sección denominada Discusión.

\begin{tabular}{|l|l|r|r|r|}
\hline \multicolumn{5}{|c|}{$\begin{array}{l}\text { Tabla 2: Correlación de las variables } \\
\text { "estado emocional", “verbal” "no verbal” }\end{array}$} \\
\hline & & \multicolumn{1}{|c|}{$\begin{array}{l}\text { estado } \\
\text { emocional }\end{array}$} & \multicolumn{1}{c|}{ no verbal } & \multicolumn{1}{c|}{ verbal } \\
\hline $\begin{array}{l}\text { estado } \\
\text { emocional }\end{array}$ & $\begin{array}{l}\text { coeficiente de } \\
\text { correlación }\end{array}$ & 1,000 & $\mathbf{0 . 5 0 0}$ & 0.221 \\
\hline & $\mathrm{N}$ & 61 & 61 & 61 \\
\hline
\end{tabular}

La Tabla 2 muestra cómo la relación entre el estado emocional y los elementos no verbales es diferente a la relación que hay entre el estado emocional y los elementos verbales. Los datos indican que la correlación entre estado emocional de Clinton y el uso de mecanismos no verbales es moderada (0.500), mientras que la correlación entre su estado emocional y el uso de estrategias verbales

1 Para analizar las posibles relaciones existentes entre estrategias verbales y estrategias no verbales, se ha aplicado el coeficiente de correlación de Pearson. Sin embargo para hallar las posibles relaciones de interdependencia entre el estado emocional de Clinton y el uso de estrategias verbales y no verbales, se ha utilizado el coeficiente de correlación de Spearman, which is a special case of the Pearson product-moment coefficient, has been selected. La razón de aplicar coeficientes de correlación diferentes se debe a la propia naturaleza de las variables, ya que la correlación Pearson requiere que las variables sean escalas (que es el caso de las etiquetas "no verbal" y "verbal"), mientras que la correlación de Spearman es una medida que se aplica con variables ordinales (como es el caso de la etiqueta "estado emocional"). 
es baja (0.221). Probablemente, una de las razones por las que puede haber esta diferencia sea que Clinton tiene más facilidad para controlar lo que dice que lo que hace. Es decir, Clinton puede llegar a ocultar con sus palabras su estado emocional, sin embargo esa tarea no es tan fácil con los elementos no verbales, ya que suelen estar emparejados a la expresión de las emociones. Quizá sea esa también la razón por la que en algunas ocasiones podamos interpretar mensajes contradictorios entre lo que Clinton expresa verbalmente y lo que transmite de manera no verbal.

Por último, se ha recurrido al uso de tablas de contingencia para valorar la relación existente entre las variables "estado emocional" (ordinal) y la variable que "privado/público" (nominal), que hace referencia al contenido o tema tratado en los turnos de palabra (Tabla 3).

\begin{tabular}{|l|c|c|c|c|}
\hline \multicolumn{4}{|c|}{ Tabla 3: Estado Emocional / Público-Privado } \\
\cline { 2 - 4 } privado/público & \multicolumn{3}{|c|}{ Estado Emocional } & \multirow{2}{*}{ Total } \\
\cline { 2 - 4 } & relajado & enfadado & colérico & \\
\hline Irrelevante & 1 & 0 & 0 & 1 \\
\hline $\begin{array}{l}\text { Privado } \\
\text { (asuntos familiares) }\end{array}$ & 8 & 0 & 0 & 8 \\
\hline $\begin{array}{l}\text { Privado } \\
\text { (escándalo sexual) }\end{array}$ & 1 & 6 & 7 & 14 \\
\hline $\begin{array}{l}\text { Público } \\
\text { (política internacional) }\end{array}$ & 0 & 15 & 0 & 15 \\
\hline $\begin{array}{l}\text { Público } \\
\text { (política nacional) }\end{array}$ & 3 & 1 & 0 & 4 \\
\hline $\begin{array}{l}\text { Privado/Público } \\
\text { (política/ escándalo sexual) }\end{array}$ & 5 & 6 & 5 & 16 \\
\hline $\begin{array}{l}\text { Privado/Público } \\
\text { (política/vida familiar) }\end{array}$ & 3 & 0 & 0 & 3 \\
\hline Total & 21 & 28 & 12 & 61 \\
\hline
\end{tabular}

En la Figura 3, a continuación, se ilustra la cantidad de turnos cuyo contenido se centran en temas de índole pública o privada en la entrevista. 


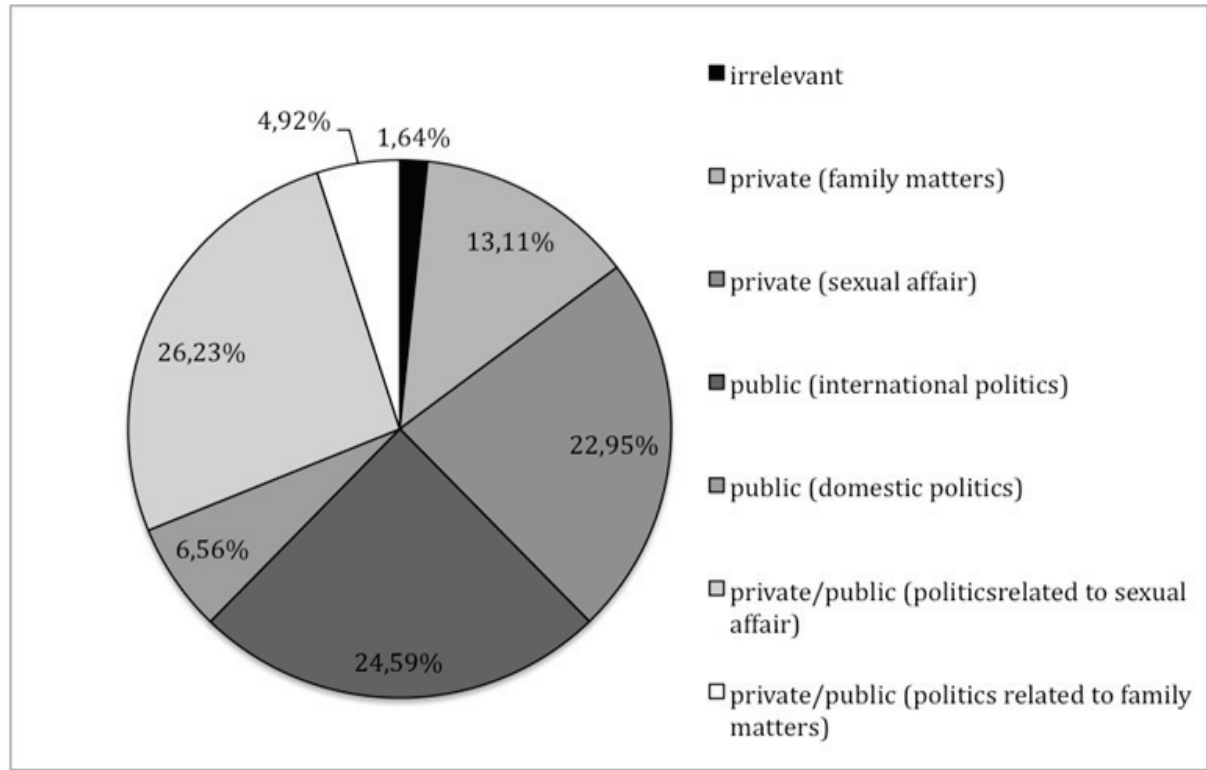

Figura 3. Contenido sobre el que versan los turnos

\section{DISCUSIÓN}

En lo que a estructura del discurso se refiere, la entrevista puede dividirse en cinco secciones diferentes, tal y como se refleja en la Figura 4.

\begin{tabular}{|c|c|c|c|c|}
\hline \multicolumn{5}{|c|}{ Entrevista } \\
\hline$\downarrow$ & $\downarrow$ & $\downarrow$ & $\downarrow$ & $\downarrow$ \\
\hline Sección 1 & Sección 2 & $\begin{array}{c}\text { Sección } 3 \\
\text { Extrema }\end{array}$ & Sección 4 & Sección 5 \\
\hline Relajación & Tensión & Tensión & Tensión & Relajación \\
\hline
\end{tabular}

Figura 4. Estructura de la entrevista

Las secciones que se indican en la Figura 4 corresponden a diferentes estadíos del estado emocional de Clinton cuando responde a las preguntas formuladas por el entrevistador. La primera sección es una etapa introductoria (etapa de calentamiento) que comprende un intervalo que va desde el comienzo de la entrevista hasta el turno Q/A8. A continuación, se muestra un clip en el que aparecen juntos Clinton y Monica Levinsky. Este clip supone una especie de puente entre la relajación de la sección 1 y la tensión de la sección 2 (desde el turno Q/A9 hasta el turno Q/A19). Posteriormente, la sección 3, que va desde el turno Q/A20 al Q/A37, es la parte de la entrevista donde hay más tensión. Esta tensión baja de intensidad en la sección 4, que empieza en el turno Q/A38 y 
termina en el Q/A56. Por último, la sección 5 corresponde de nuevo a una etapa de relajación y comprende desde el turno Q/A57 hasta el Q/A61.

En lo que a comunicación no verbal se refiere, se ha podido comprobar que muchos de los elementos no verbales que están presentes en la entrevista no son ciertamente estrategias comunicativas que se utilicen con el fin de convencer a la audiencia o simpatizar con ella, sino que más bien se trata de elementos que parecen no estar controlados por el emisor, Clinton, y que reflejan sus sentimientos o emociones. La razón por la que afirmamos que estos signos no verbales parecen estar fuera del control del emisor es que en la mayoría de los casos en los que aparecen transmiten un mensaje bien distinto del que transmiten las palabras del emisor. Es decir, el emisor se esfuerza por codificar un mensaje verbal que quiere transmitir a la audiencia, sus palabras comunican tranquilidad, calma y control, pero sus gestos comunican algo diferente, transmiten nerviosismo, tensión y descontrol. No obstante, los signos no verbales también se utilizan en esta entrevista como estrategias comunicativas y, por lo tanto, como recursos controlados por el emisor con el fin de conseguir unos objetivos concretos.

Dentro de la comunicación no verbal, nos detenemos primero en el estudio del paralenguaje. Por paralenguaje nos referimos a las cualidades y modificadores vocales tales como ritmo, intensidad, volumen, tempo, tono, etc., así como a las vocalizaciones o diferenciadores vocales tales como tos, risa, llanto, bostezo, etc., que pueden acompañar al habla o que se pueden producir de manera aislada y que aportan información relevante sobre los participantes de la interacción. Harris y Rubinstein (1975:263) señalan que nuestra voz es:

...an unusually sensitive barometer of how we feel, the particular "mood" we are in at the moment of speaking.... We learn to exude expression of this kind and to perceive it in the speech of others according to a set of shared expectations prescribed by our culture. The basis of most of our intuitive impressions of this kind often turns out to be some conventional combination of the use of pitch, loudness, tempo, and duration.

Tras tener en cuenta factores tales como el tono, volumen, tempo y duración y analizar su uso a lo largo de la entrevista, hemos considerado que el turno Q/ A29 es el que representa mayor tensión. En este turno, no es necesario que entendamos las palabras de Clinton para que percibamos sus sentimientos de ira y furia. El entrevistador ha estado interrogando a Clinton sobre su aventura sexual con Monica Levinsky desde el turno Q/A13 y, aunque se podían apreciar signos no verbales que mostraban su malestar, al menos sus palabras conseguían comunicar una aparente calma tensa. Sin embargo en el turno Q/A29 Clinton ya no puede seguir ocultando sus sentimientos: 
Clinton: Wasn't as I saw it sir, we had several years of evidence. We had several years of evidence. Kenneth Starr would not be allowed to be prosecutor against me as a defendant in any decent court in the land.

Dimbleby: You obvious[ ly ]

Clinton: [And], and let me just say this. One of the reasons he got away with it is because people like you only ask people like me the questions. You gave him a complete free ride (...)

Aparentemente, Clinton ha completado su turno afirmando "Kenneth Starr would not be allowed to be prosecutor against me as a defendant in any decent court in the land" y, por lo tanto, ahora es el turno del entrevistador, Dimbleby. Sin embargo, en contra de nuestras expectativas, Clinton cambia de opinión e interrumpe al entrevistador, que ya había comenzado su turno. Clinton levanta la voz y utiliza un tono elevado porque ya no puede seguir ocultando sus sentimientos más profundos de ira y rabia. Estos signos paralingüísticos son un reflejo de su malestar, no tanto con Kenneth Starr, sino con Dimbleby por seguir insistiendo en este tipo de preguntas relacionadas con el escándalo sexual. Es obvio que Clinton sabía que este tema se iba a tratar y, seguramente, se habría preparado para salir airoso de estas incómodas preguntas, como lo demuestra el hecho de que incluso conteste con humor en algunas situaciones difíciles. Pero ese control y dominio verbal que demuestra en numerosas ocasiones contrasta con su comportamiento no verbal, que probablemente no puede controlar y de ahí que explote.

Si bien anteriormente hablábamos de un uso descontrolado de los signos no verbales, hay otras ocasiones en las que los elementos no verbales son utilizados de una manera controlada por Clinton como estrategias comunicativas. Por ejemplo, habla de manera más pausada o reduce la velocidad con los siguientes fines: (1) para tener tiempo de pensar en lo que va a decir a continuación $(\mathrm{Q} /$ A47); (2) para dar énfasis a sus palabras (Q/A49); (3) para dar la impresión de que está relajado (Q/A6), etc.. En el turno Q/A47, el entrevistador formula un pregunta difícil o comprometedora a Clinton (sobre si aconsejó a Tony Blair que no apoyara al Presidente Bush). Primero hay un silencio y luego un retardo lingüístico ('linguistic delay'), y aunque articula algunas palabras ("Well I have sa.. I don't. you're asking me a question and I'm not sure exactly ...") no dice absolutamente nada. Este retardo lingüístico se produce de manera muy pausada:

Clinton: Well I have sa.. I don't.. you're asking me a question and I'm not sure exactly when I was at Chequers, vis a vis the Iraq date. I've been there several times since I left office. Tony Blair and I are friends...

En el intercambio comunicativo Q/A49 (todavía hablando sobre su relación con Tony Blair y la invasión de Irak) Clinton aminora la velocidad con la que habla para enfatizar determinadas palabras. De hecho, las palabras que quiere 
enfatizar son de tal importancia para él que incluso vuelve a repetirlas a continuación dos líneas más abajo:

Clinton: But here's the problem Tony Blair faced. Blair had a problem unique in Europe and that's why I went to the Labour Party Conference in Blackpool and defended him ...he had a problem unique in Europe (...)

Como se ha mencionado anteriormente, otra de las razones por las que Clinton utiliza la estrategia de hablar de manera más pausada es para dar la impresión de que está relajado (Q/A6). En esta ocasión, en el turno Q/A6, está hablando de ira pero quiere hacer creer a la audiencia que él no está enojado y que no es una persona que suela enfadarse y por ese motivo habla muy despacio, que es una manera de mostrar su calma:

Clinton: Well I think whenever you're, the, the Greeks said once, Those whom the gods would destroy they first make angry. If you go round mad you can't, you don't think very well, and you wind up doing things that you shouldn't do. And I think there are numerous points in my life, where I really was angry and I, it bothered me. I also think a lot of anger is quite healthy and I've bent over backwards because I tried to be a peace maker in my home; I bent over backwards not to be angry, and never to show anger and I think there's a price for that as well.

En varias ocasiones a lo largo de la entrevista, Clinton hace uso de un tipo de vocalización paralingüística, la risa, con la intención de mitigar o suavizar una situación que no es agradable (generalmente porque Dimbleby le está haciendo preguntas que le incomodan). Por ejemplo, se puede apreciar esta vocalización cuando el entrevistador le pregunta sobre cómo se sentía teniendo que enfrentarse con el problema de Al Qaeda y al mismo tiempo teniendo que dormir en el sofá porque le habían echado del lecho conyugal. Clinton es consciente de que el entrevistador pretende demostrar que él no estaba capacitado en ese momento para resolver el terrible problema del terrorismo por estar envuelto en otros problemas de su vida privada. Entonces Clinton se ríe, suponemos que no porque le haga gracia la situación, sino porque es una manera de amortiguar o aliviar la incomodidad que le produce lo que está diciendo Dimbleby (A40). En algunos casos, lo que encontramos es una risa nerviosa, como sucede en los turnos A34 y A35. Lo que resulta interesante es que esos tres casos de uso de la risa como estrategia mitigadora (A34, A35 and A40) ocurren en turnos considerados de tensión o tensión extrema.

Por medio de los signos paralíngüísticos podemos conocer el estado emocional del hablante, su actitud y sus sentimientos, podemos saber el tipo de relación que tiene con los otros participantes de la interacción comunicativa, así como de 
la intención del hablante de continuar hablando o de finalizar el turno, etc. En palabras de Bolinger (1986:338):

Intonation is important for who is speaking, for who will be taking the next turn, for how the act is to be understood (explanation, apology, challenge), for how the speaker will be evaluated (as an individual, as a native speaker, as a member of a given social class) - to mention only a few of the things that affect our roles as speakers and listeners.

De la misma manera que la voz y sus cualidades son importantes en el proceso comunicativo, su ausencia es también relevante. Se pueden distinguir dos tipos diferentes de situaciones en los que hay ausencia de voz: los silencios, que son espacios de tiempo en los que el hablante no emite voz o ruido alguno (denominados por Knapp 1980 pausas vacías - 'unfilled pauses'); y las pausas (denominadas por Knapp 1980 'filled pauses'), que son espacios de tiempo ocupados con alternantes (uh-huh, ah, oh, ooh, shh, mmmh, eh, etc.) o caracterizadores (llanto, tos, risa, etc.). Knapp (1980:222) analiza las pausas de la siguiente manera:

... the two major types of pauses are the unfilled pause (silent) and the filled pause. A filled pause is simply filled with some type of phonation such as "um," "uh," stutters, false starts, repetitions, and slips of the tongue. A variety of sources associate filled pauses with a range of generally undesirable characteristics. Some people associate filled pauses and repetitions with emotional arousal and others feel that filled pauses may reduce anxiety, but jam cognitive processes.

Como ya se ha mencionado, el ritmo comunicativo de Clinton se ralentiza en el turno Q/A47 con la finalidad de tener tiempo para pensar en lo que va a decir. Se produce una pausa vacía (es decir, un silencio) y luego hay dos pausas ocupadas (que suponen una especie de falsos comienzos de turno). El entrevistador le había hecho una pregunta difícil o comprometedora (si había recomendado a Tony Blair que no apoyara al Presidente Bush) y Clinton necesitaba tiempo para pensar qué contestar.

En lo que a elementos no verbales visuales se refiere, los gestos faciales son los más obvios o fácilmente perceptibles. La cara es probablemente una de las fuentes de información no verbal más notables para los participantes de una interacción, ya que siempre está visible. Según Richmond, McCroskey y Payne (1991:73):

The area of the face, and in particular the area around the eyes, probably is the most significant area of the body for communicating nonverbal messages. If research has taught us anything, it has helped us to understand that the human face is the primary tool used for transmitting expressions of emotion. The facial muscles can provide such a complex repertoire of configurations that most 
of us, if we worked at it, could manage to put our faces into more than a thousand different appearances.

Uno de los mecanismos faciales que los políticos suelen usar con bastante frecuencia como estrategia comunicativa es la sonrisa. La sonrisa puede ser un indicador de alegría pero también puede utilizarse con otros fines². A lo largo de la entrevista, se ha podido comprobar que las sonrisas de Clinton tienen un objetivo fundamental: enmascarar u ocultar su enfado, malestar y nerviosismo. Al principio de la entrevista Clinton estaba más relajado, pero a partir de las preguntas sobre el escándalo con Monica Levinsky, Clinton tiene que empezar a luchar para no dejar escapar sus emociones. Hace un gran esfuerzo por dar la apariencia de una persona tranquila y sosegada pero su cara no comunica el mismo mensaje. A este respecto, Ekman, Friesen y Ellsworth (1972:2) arguyen:

Although smiles may be a reliable index of pleasure or happiness, a person may also smile to mask a feeling he wishes to conceal or to prevent a feeling when he has no emotion at all. Is the face like an involuntary system or is it subject to voluntary activation and thus vulnerable to purposeful control and disguise? Clearly, it is both.

En lo que se refiere a la postura, este elemento no verbal aporta información importante sobre la actitud de Clinton. Según Mehrabian (1972), hay dos dimensiones de la postura que están asociadas a la comunicación de la actitud: there are two dimensions of posture which are associated with communication of attitudes: el factor de inmediatez y el de relajación: "Forward lean is suggestive of greater immediacy, as in the presence of someone liked. Asymmetry of arms and legs plus sideways and backwards lean is indicative of relaxation that may be present, for Americans, with another of lower status. While these dimensions may have cross-cultural validity, it has not been clearly shown that the postural variables indicative of a particular dimension are universal." (citado en Ramsey 1979:120). Cuando Clinton se inclina hacia delante en la entrevista, o bien quiere manifestar su atención a lo que Dimbleby le está preguntando, o bien quiere intimidar al entrevistador por el tipo de pregunta que le está formulando. Una postura típica de Clinton es la de apoyar la mano en la barbilla, que suele utilizar para indicar que está prestando atención a lo que le dice el entrevistador. En esta entrevista, se han encontrado también dos variantes de esta postura: (1) agarrarse impacientemente la barbilla, que se ha utilizado en

2 Algunos investigadores han señalado que las sonrisas pueden ser de diferentes tipos, y que implican el movimiento de músculos diferentes. Por ejemplo, cuando se produce una sonrisa que expresa alegría, se activa el músculo cigomático y los músculos que rodean los ojos (obicularis oculi). Sin embargo, cuando la sonrisa se utiliza para enmascarar u ocultar otros sentimientos, se activa el músculo cigomático pero esta vez lo hace junto con otros músculos que también se activan en la expression asco, miedo o tristeza. Para un análisis más detallado sobre este aspecto pueden consultarse las obras de Ekman, Friesen, y O'Sullivan (1988) y Duchenne (1862/1990). 
momentos de tensión extrema (Clinton intenta controlarse) y (2) apoyar la mejilla en la mano, que es la postura que se ha utilizado cuando Clinton se siente agotado, después de un episodio de tensión o agitación.

El contacto visual es también un factor noverbal importante en el proceso comunicativo. Principalmente se utiliza para regular la comunicación pero también es un indicador de dominio y poder. Cuando Clinton está muy enfadado (tercera sección), mira al entrevistador con desdén e incluso desprecio (A29). Esta manera de mirar a Dimbleby nos informa sobre la relación de Clinton con el entrevistador y su actitud hacia él.

Ahora vamos a proceder al análisis de las estrategias verbales encontradas en esta entrevista.

Lista de tres: Se trata de un recurso retórico muy utilizado como estrategia persuasiva por un buen número de políticos. Clinton lo utiliza en numerosas ocasiones en esta entrevista. Justo antes del siguiente extracto el periodista le ha preguntado a Clinton el motivo por el que él le ofreció "en bandeja" a la oposición una buena razón para atacarle: el asunto Lewinsky (líneas 198-203).

Clinton: Well I, I tried to explain that. I, it, it happened under circumstances in which people who had lived parallel lives become quite vulnerable. It happened at a time when I was angry, I was under stress, I was afraid I was going to lose my fight with the Republican Congress...

Clinton explica los motivos que le condujeron a actuar de esa manera y tiene que resultar convincente. Por ese motivo, utiliza una lista de tres: "I was angry, I was under stress, I was afraid”. Para llamar la atención sobre esta lista él incluso utiliza los dedos para numerarlos. En relación a este tipo de estrategia Atkinson (1984:57) explica que:

"In speeches, conversations and most other forms of communication, the most commonly used type of list contains three items, and an example of such a list has just been used to start this sentence. One of the main attractions of threepart lists is that they have an air of unity or completeness about them. Lists comprising only two items tend to appear inadequate or incomplete."

Tres es el número mínimo necesario para ofrecer un patrón de algo. Si se nos dan dos elementos de una secuencia (3/9/?), uno puede adivinar más o menos el tercero: 27 (si el número anterior es multiplicado por tres) ó 15 (si al número anterior se le suma 6) u 81 (la raíz cuadrada del número anterior). Sin embargo, si se nos da el tercero (3/9/81) uno puede establecer el patrón con total seguridad (la raíz cuadrada del número anterior).

Extreme Case Formulations (ECF): Fue Pomerantz (1986) quien introdujo este término por vez primera y Edwards (2000:347-8) quien explica que: “... 
descriptions or assessments that deploy extreme expressions such as every, all, none, best, least, as good as it gets, always, perfectly, brand new, and absolutely." Clinton los utiliza con bastante frecuencia en esta entrevista cuando él quiere indicar que algo es totalmente decisivo y definitivo.

Cuando a Clinton se le pregunta por el comportamiento de su familia después del asunto Lewinsky, él explica que aunque "he was getting a whipping at home" en realidad se lo tenía merecido. Esta respuesta es estratégica en el sentido que se trata de una manera de reconocer públicamente su error. Clinton utiliza un ECF para reforzar aún más el reconocimiento de su culpa:

CLinton: Oh actually I thought it was healthy, I thought that in a funny way I thought the fact that I was sleeping on the couch and they were still in the same house with me meant that Hilary and Chelsea hadn't given on me... I thought whatever they wanted to say or do to me, Hilary and Chelsea, they had an absolute right to do so the fact that I was still able to stay under the same roof does...

Este ECF es simultáneo a un gesto que utilizamos cuando queremos indicar que algo es definitivo: con las palmas hacia abajo movemos las manos hacia la derecha y hacia la izquierda. Este gesto refuerza aún más el efecto persuasivo de este ECF.

Pomerantz (1986: 227) resume de la siguiente manera los principales usos de los ECFs:

(1) to assert the strongest case in anticipation of non-sympathetic hearings,

(2) to propose the cause of a phenomenon,

(3) to speak for the rightness (wrongness) of a practice.

Sin embargo, hay que señalar que estos ECFs son muy débiles en el sentido que es muy fácil refutarlos. Si alguien menciona simplemente un contra-ejemplo, la validez del ECF queda cuestionada. Por este motivo, es muy frecuente encontrar una versión debilitada ("a softener") justo después del ataque al ECF. La secuencia sería: ECF - desafío - versión debilitada.

También hay que aclarar que, a pesar de esta "debilidad" de los ECFs, en un estudio llevado a cabo por Edwards (2000) se descubrió que el uso de los ECFs es muy popular y está muy difundido en la sociedad. El motivo de este comportamiento es que en la mayoría de las ocasiones las personas aceptan y admiten sus interpretaciones no literales.

$D P C C$ : Otra de las estrategias utilizadas por Clinton en esta entrevista es la que se conoce como DPCC “dos premisas controlan la conclusión”. Esta estrategia está relacionada con la manera en la que se estructura la información y consta de dos fases: primero, se presenta una situación difícil de resolver y, una vez la 
audiencia se ha dado cuenta que la situación sería difícil para cualquiera, en segundo lugar el entrevistado revela que él ha sido capaz de solucionarla:

Fase 1 (algo es difícil) + Fase 2 (Yo lo puedo hacer)

Obviamente, el entrevistado podría haber utilizado una estructura más sencilla y simplemente haber dicho que él ha sido capaz de solucionar una situación difícil.

Como se puede observar, esta estrategia está basada en la lógica. $\mathrm{Si}$ (1) algo es difícil de resolver y (2) yo puedo resolverlo, entonces ¿cuál es la conclusión que se extrae de las dos premisas anteriores? Que yo soy muy bueno. Esta conclusión no se menciona explícitamente en ningún momento pero el entrevistado intenta que la audiencia llegue a ella por sí misma.

En esta entrevista a Clinton se le pregunta si el asunto Lewinsky hizo mella en su capacidad de concentración a la hora de tomar el tipo de decisiones que un Presidente debe tomar. Aunque él afirma que no, el entrevistador le pregunta "Do you yourself feel that?" y Clinton responde:

Clinton: Absolutely. But I tell you this is quite interesting and when I talk about the parallel lives thing when I was, you know, as a child and you pointed out that .. some of the problems with having parallel lives and I agree with you which I tried to be candid about... And when they needed me I spent a few minutes and answered their questions so they briefed me and for the rest of the time we worked on the Presidency and it sounds crazy to someone who's never been through it but I knew how to do that. And I'd had a lot of practice in the ninety two campaign in the first term but, basically, it went back to my childhood for learning to live with big pot of problems. When my mother wrote her memoirs she talked about the same thing.

Mientras pronuncia este DPCC, Clinton abre sus manos con la finalidad de resultar sincero, claro y preciso.

Paralelismo (Repetición): Algunas veces Clinton utiliza estructuras paralelas para reforzar una idea. O bien repite la misma estructura, o bien las mismas palabras. Al hablar de la repetición Cockcroft and Cockcroft (1992:131) explican que "This is probably the major resource of schematic rhetoric and the one with closest affinity to the spontaneous expression of emotion."

En el siguiente extracto Clinton desea enfatizar que él estaba peleando contra enemigos políticos durante el juicio Lewinsky. Uno de estos enemigos era Kenneth Starr e insiste en el hecho de que las pruebas que apoyan lo que él está diciendo las conoce todo el mundo porque pertenecen al pasado (líneas 310-312): 
Clinton: Wasn't as I saw it sir, we had several years of evidence. We had several years of evidence. Kenneth Starr would not be allowed to be prosecutor against me as a defendant in any decent court in the land.

Para reforzar esta repetición el señala hacia atrás como refiriéndose a un suceso del pasado.

Según Cockcroft and Cockcroft (1992:131): "The pattern created by a repeated word, or the rhythm created by a repeated phrase, validate Coleridge's remarkable insight about 'striv(ing) to hold in check the workings of passion's."

Formulaciones Esquemáticas (Script Formulations): Una estrategia persuasiva muy utilizada consiste en hacer creer al otro que compartes las mismas ideas y sentimientos. Éste es el principal objetivo de las denominadas formulaciones esquemáticas (script formulations) (Edwards 1994, 1995, 1997): to construct a common socio-psychological reality based on a set of beliefs and experiences shared by speaker and listener. Edwards (1995) menciona una serie de recursos esquemáticos gramaticales que tienen como finalidad describir una formulación esquemática: tiempos verbales de presente con valor iterativo, el uso del modal "would", expresiones como "you know", etc. Cuando una persona utiliza estas formulaciones esquemáticas en su discurso, él/ella quiere mostrar que no se trata de algo fortuito sino más bien algo ya conocido y con un patrón que se puede preveer.

Clinton utiliza este tipo de estrategia en varias ocasiones. Por ejemplo, él utiliza dos formulaciones esquemáticas en el turno 13. El entrevistador le acaba de decir que él le ha ofrecido a sus enemigos un regalo con el asunto Lewinsky. Clinton contesta que ya le han hecho esa pregunta antes y por eso utiliza una formulación esquemática como "But you know when people ask me this question”. Esto significa que él ya está acostumbrado a este tipo de preguntas y esto les puede llevar a pensar al entrevistador y a la audiencia que va a ofrecer una buena contestación. Unas líneas más adelante él usa la expresión "And you know" en un intento por sonar cercano y conseguir una especie de "alianza" con el entrevistador porque Clinton sabe que va a comenzar un asunto delicado (i.e. el asunto Lewinsky).

Clinton: Of course I did, and was it rational? No. So I do my very best to explain why I think it happened. But you know when people ask me this question, well how could you do something so stupid, when you knew they were after you. Well of course, if I'd been thinking straight, I wouldn't have done it. If - but I hope that you and everyone else who asks me this question, never has to know what it's like to have somebody who despises you be given unaccountable legal power, to indict the innocent, because they will not lie and to

3 S. T. Coleridge, Biographia Literaria, Ch. XVIII - e.g. Everyman's Library edn, ed. G. Watson (London: Dent, 1956) p. 206. 
exonerate the guilty because they will, and then to be treated as a totally legitimate person in the press, as if obviously you must have done something wrong or why are they doing all this? And you know, it's hard to think straight when that's going on.

Según Edwards (2003: 38) “... formulating events as regular makes them both factually robust and also somewhat knowable in advance without having to wait and see for any specific instance. Script formulations are presented as if based on lots of instances, and perhaps lots of people's repeated (consensual) experiences of instances.”

Uso de los pronombres: Clinton también utiliza de manera estratégica los pronombres personales. Por ejemplo, en las líneas 198-203 Clinton explica que Starr había convertido un asunto privado en un asunto legal. Entonces, el entrevistador le pregunta si piensa que eso estaba mal y Clinton responde "Of course". Justo después de eso tiene lugar el siguiente intercambio (líneas 209-218):

Dimbleby: Did you think it was dangerous at the time?

Clinton: What they were doing?

Dimblebs: What you were doing. Did you think it was risky?

Clinton: I don't know that I, I don't - I can't answer that. I don't know what I thought about it. (interjects) It didn't last very long and ... and the accounts are not entirely accurate of what did happen; so I don't want to talk about that. I've said, all I have to say about that in the book. I'm not saying any more about that.

Está claro que Dimbleby le está preguntando a Clinton si lo que él estaba haciendo era peligroso pero él finge no haber entendido la pregunta. Él intenta evitar esa pregunta porque no le agrada. De hecho, cuando él contesta a la pregunta retira la mirada y muestra un rechazo total hacia ella. Clinton opina que se trata de una pregunta muy personal (utiliza hasta 10 veces el pronombre personal "I") y discrepa (usa 7 negaciones).

Preguntas retóricas: Las preguntas retóricas son muy estratégicas porque elevan el nivel de conciencia que una persona tiene de algo. Clinton sabe esto y por este motivo las utiliza muy frecuentemente. Es especialmente interesante el siguiente extracto (líneas 446-456):

Clinton: Well because, I mean in theory we could have but we would have been all alone everybody would have thought we were crazy based on that. And then could I have.. would I have done more after the USS Cole in October two thousand. And could I have if, that's one big if. If the government intelligence agencies in this case the FBI and the CIA had agreed with me even though my term was almost over and had told me that they agreed for 
sure that Bin Laden and Al Qaeda were responsible for the USS Cole, a finding they did not make until after I had left office, I would have done more then. Would it have succeeded in getting Bin Laden, would it have prevented 9/11, I don't know. I mean, we've got.. look how long we've been in Afghanistan and we still haven't succeeded in that. And believe me, I have asked a lot of these questions myself.

Antes de realizar esta pregunta, el entrevistador le había dicho a Clinton que le había dado una prioridad baja al asunto del terrorismo y la lucha contra Al Qaeda. Clinton usa cuatro preguntas retóricas (todas ellas con verbos condicionales: would, could, would y would) para mostrar que nadie puede saber qué habría ocurrido y por ese motivo nadie puede culparle por nada.

Zillman (1972:161) resume el uso estratégico de este tipo de preguntas de la siguiente manera:

It may be argued that the assumed covert agreement response elicited by a rhetorical agreement question, as compared to the relatively passive decoding of an assertion in statement form, raises the individual's level of awareness. It makes him cognizant of his position on a particular issue, and it may activate issue-related cognitions to consolidate and bolster his evaluations, thereby facilitating his involvement with the issue, and possibly producing some self-commitment.

Opuestos (Contrastes): Otra estrategia usada por Clinton está relacionada con la manera de presentar un asunto. Al entrevistador y a la audiencia se les muestran las dos caras de la moneda, no hay término medio. En el siguiente extracto (líneas 145-162) se puede leer cómo al entrevistador y a la audiencia se les muestran alternativas positivas contra negativas:

Clinton: Yeah, but I, I don't like that because that exonerates everybody else of responsibility for the decisions that they make. The New Right that controlled the Republican Party in Washington and the political press had the same interests. They thought it was all about power, I thought it was about how power was used. I was interested, to me, the way I kept score in my Presidency was, Did more people have jobs or not? Did more people move out of poverty or not? Did the crime rate go down or not? Were more kids breathing clean air and fewer getting asthma? What was our record in the world? Did we advance peace and prosperity and security or not? That's how I kept score. Others kept score in a totally different way. You know, are we hurting the other side or not? Have we got a good story today that is about personal destruction?...

Justo antes de esta pregunta Clinton había visto un video sobre Monica Lewinsky y él sabe que le van a preguntar sobre ella. Él intenta centrar su respuesta es asuntos estrictamente políticos y es por esto que hace un despliegue 
de todos sus logros políticos en un intento por contrapesar lo que él sabía que le iban a preguntar (su metedura de pata sexual). Clinton utiliza preguntas retóricas disyuntivas en las que el entrevistador y la audiencia se ven forzados a elegir una sóla opción, no hay otra alternativa.

Comparaciones: En algunas ocasiones Clinton utiliza comparaciones para realzar sus éxitos. En la siguiente respuesta (líneas 87-94) él se compara con el resto de los presidentes y defiende que él es diferente por su sentido de la lealtad, apoyo y determinación (lista de tres) a sus amigos personales:

Cuinton: (interrupts) It, if, when you live ... my life has been both selfish and selfless. I mean if you live the kind of life I live, I've lived, you're running for office - it's almost impossible, as I say in this book, I may be the only person who got elected President ever, because of the loyalty, support and determination of his personal friends, who just wouldn't let my campaign die.

Metonimia: Cuando no es muy conveniente dirigirse a algo directamente, Clinton utiliza la metonimia, que consiste en la sustitución de una palabra por otra diferente que guarda alguna relación con la primera.

En 1999 Clinton y Blair bombardearon Iraq durante cuatro días. En lugar de eso, Clinton dice:

Clinton: Yes. For most of the time I was there, the idea was that his military is less than half the strength it was in the first Gulf War, which is factually true...Then in ninety eight when Saddam kicked the inspectors out to try to force us to lift the sanctions. Prime Minister Blair and I bombed him for four days and we bombed the sites where thought the chemical and biological materials would be...

No es conveniente para Clinton admitir que bombardeó un país lleno de gente inocente, en lugar de eso dice que bombardeó a Saddam.

Cockcroft and Cockcroft (1992:120) explican de esta manera el uso estratégico de la metonimia:

Through a common association, or 'compact reference' (Nash, Designs, p. 55) within the minds of author and audience, an idea put into words metonymically represents unexpressed or implicit ideas and associations. This will have obvious implications for persuasion in general, and advertising in particular.

Dilaciones: Cuando Clinton se ve atrapado entre la espada y la pared se toma su tiempo para responder. En un extracto anterior (líneas 446-456) Clinton no comienza a responder a la pregunta hasta la palabra número 11: 
CLINTON: Well because, I mean in theory we could have but we would have been all alone everybody would have thought we were crazy based on that. And then could I have...

Le habían preguntado porqué él no invadió Afghanistan apoyándose en los bombardeos a la Embajada Africana. Es muy pregunta muy delicada y por ese motivo se dilata su respuesta. Es interesante aquí mencionar el uso de "well". Schiffrin (1987) sugiere que un individuo utiliza "well" casi de manera exclusiva al comienzo de una respuesta para señalar que lo que viene a continuación puede que no sea del total agrado del oyente. Ejemplos (Byron and Heeman, 1997) $)^{4}$

utt16 u: how long would it take to load the oranges from the warehouse into the engine

utt17 s: uh well we can't load oranges into an engine we need a boxcar

Según Byron y Heeman (1997), en los diálogos de Trains “well” es

... typically used to correct a misconception or to suggest an alternative plan.It is found not only at the beginning of responses, but also after the other speaker has just stated a fact or drawn a conclusion with which the current speaker is about to disagree.

Ellos proponen el siguiente ejemplo:

utt53 u: and then I'm done

utt54 s: well you have to get to Avon still

Byron and Heeman (1997) dicen que cuando un individuo comienza un turno con "well" eso nos hace pensar que el hablante va a expresar un desacuerdo o bien va a corregir alguna información (implícita o explícitamente) contenida en el turno anterior.

De igual manera, Schiffrin (1985) también defiende que "well" precede una respuesta no muy satisfactoria y le atribuye el papel de marcador de coherencia (Schiffrin, 1985:650):

The results thus far suggest that well is more likely to be used when respondents cannot easily meet conversational demands for response because the informational content of their response will not fit the coherence options just opened by a prior referent.

${ }^{4}$ Donna K. Byron y Peter A. Heeman "Discourse marker use in task-oriented spoken dialog" en Proceedings of Eurospeech'97. Se puede encontrar un informe más detallado en http://www. cs.rochester.edu/trs/ai-trs.html. (Página consultada el 25-05-2010). 
En este estudio, Schiffrin revela que raramente una pregunta tajantemente positiva o tajantemente negativa están precedidas por "well". Sin embargo, casi la mitad de las respuestas "no claras" a preguntas del tipo sí/no estaban precedidas por “well'. De manera similar, cuando una respuesta a una pregunta del tipo wh- no ofrecía la información solicitada se encontró una tendencia clara a ser precedidas por "well".

Aunque por razones de exposición en algunos casos se ha hecho referencia a determinadas estrategias verbales y no verbales de manera aislada, es importante señalar que la comunicación es un proceso multicanal en el que intervienen tanto mecanismos verbales como no verbales en sincronía. Como se ha podido apreciar en la sección de resultados, después de calcular el ceficiente de Pearson para las variables "verbal" y "no verbal", existe una alta correlación positiva entre los dos tipos de elementos (0.795). Esto implica no sólo que los recursos verbales y no verbales tienden a aprecer juntos, sino que además su uso aumenta o disminuye a la vez. A primera vista, esta afirmación parece lógica y, en consecuencia, podemos interpretar que Clinton utiliza tanto estrategias verbales como no verbales para lograr sus objetivos. Sin embargo, si analizamos el significado y la función de los elementos en detalle, se puede obsevar que el hecho de que los signos verbales y no verbales se den conjuntamente no implica que coincidan en significado y función. De hecho, se dan dos situaciones diferentes:

1. Las palabras y los gestos coinciden en significado y función.

2. Las palabras y los gestos presentan significados $y / o$ funciones diferentes.

La primera de las opciones es la que se ha señalado anteriormente como una situación lógica, ya que implica que Clinton tiene un objetivo en mente y para lograrlo hace uso de todo tipo de estrategias, verbales y no verbales, todas ellas con un objetivo común y, por lo tanto, comunicando mensajes acordes. Un ejemplo de esta situación puede apreciarse cuando Clinton está hablando de ira ("anger") y se esfuerza por demostrar que él no es una persona que esté enfada o irritada. Para conseguir este objetivo, Clinton utiliza, por un lado, una estrategia verbal (el contraste de conceptos) y, por otro lado, una estrategia no verbal (hablando más despacio para dar la impresión de que está relajado). En consecuencia, se puede decir que en este caso los mecanismos verbales y no verbales coinciden en significado y función, y se puede pensar que esta situación no es una mera coincidencia, sino que Clinton ha hecho uso de estos recursos de manera consciente e intencionada para lograr el fin que tenía en mente.

La segunda situación que se mencionaba es completamente diferente porque implica que las palabras de Clinton no comunican lo mismo que sus gestos. En esta ocasión, Cinton utiliza diferentes estrategias verbales con un objetivo determinado pero los recursos no verbales que co-ocurren con las estrategias verbales difieren en significado, pudiendo incluso contradecir lo que se ha expresado 
verbalmente. Ante esta situación se puede pensar que los elementos no verbales en cuestión no forman parte de la estrategia de Clinton, sino que más bien son elementos no controlados que el entrevistado no ha sido capaz de refrenar $\mathrm{u}$ ocultar. Por ejemplo, en el turno Q/A36, Clinton desea sonar convincente y definitivo cuando responde "no" pero, al mismo tiempo, se puede observar que no mantiene contacto visual alguno con el entrevistador y que, por lo tanto, evita encontrarse con la mirada de Dimbleby. Esta falta de concordancia o afinidad entre las palabras y los gestos de Clinton llevan a concluir que el entrevistado pueda estar mintiendo. Una situación similar ocurre en el turno Q/A42 en el que Clinton recurre a un gran número de preguntas retóricas para sonar convincente pero su discurso demasiado lento, así como el alargamiento excesivo de vocales, muestran que en realidad no sabe qué contestar o qué decir.

Como ya se ha mencionado con anterioridad, el uso de mecanismos no verbales de manera no controlada por parte de Clinton parece estar en estrecha relación con su estado emocional. Por medio del coeficiente de Spearman, se ha podido comprobar que hay una correlación moderada entre el uso de signos no verbales y el estado emocional (0.500). Consideramos que una de las razones por la que esta correlación no es más alta pudiera ser por su propia condición de político, ya que, aunque sus gestos son un barómetro de sus sentimientos, como buen político probablemente es capaz de ejrcer algún control sobre ellos.

La principal causa del estado emocional de Clinton se encuentra en las preguntas formuladas por el entrevistador. La correlación de contingencia entre las variables "contenido público/privado" y "estado emocional" ha demostrado ser alta (0.687). Clinton se enfada mucho más cuando las preguntas entán relacionadas con el asunto Monica Lewinsky. La relajación, sin embargo, tiende a manifestarse en los turnos en los que Clinton responde a preguntas sobre su vida privada familiar. En algunos turnos se mezclan los asuntos de la vida privada de Clinton con los asuntos de su vida pública. En el turno Q/A33 Dimbleby sabe que Clinton ha perdido los nervios con las preguntas anteriores sobre su escándalo con Mónica Lewinsky y, como es habitual en todo entrevistador, Dimbleby quiere seguir esta línea porque es conocedor del atractivo y repercusión que estas situaciones generan en la audiencia. Por otra parte, el entrevistador ha comunicado a Clinton que no pretende estar hablando toda la entrevista sobre este asunto ("not intend to talk endlessly about her"), así que introduce un tema nuevo relacionado con la vida pública de Clinton, Al Qaeda. El ingenio de Dimbleby consiste en relacionar las dos vidas del entrevistador, intentando descubrir en qué medida la vida privada de Clinton podía haber afectado en las decisiones que había de tomar en su vida pública.

Los datos obtenidos en el presente estudio demuestran que el uso de recursos no verbales está directamente relacionado con el estado emocional del entrevistado (y no siempre de acuerdo con lo que el entrevistado dice). Además, se ha podido comprobar que el estado emocional de Clinton está determinado por la 
naturaleza de las preguntas formuladas por el entrevistador. Por último, también se ha podido apreciar una correlación positiva entre el uso de estrategias verbales y el uso de mecanismos no verbales, que implica que el uso de ambos mecanismos aumenta y disminuye a la vez de manera simultánea.

\section{REFERENCIAS BIBLIOGRÁFICAS}

Alan, B. (1991). The Language of Newws Media. Oxford: Blackwell

Atrinson, R. (1984). Our Masters' Voices: The Language and Body Language of Politics, New York, Methuen.

Atrinson, J. M. y P. Drew (1979). Order in Court, Oxford Socio-Legal Studies, Atlantic Highlands, NJ: Humanities.

Blum-KulKa, S. (1983). “The dynamics of political interviews". Text, 3, 131-153.

Bolinger, Dwight (1986). Intonation and its Parts, Stanford, California, Stanford University Press.

Bull, P. (1994). "On identifying questions, replies, and non-replies in political interviews". Journal of Language and Social Psychology, 13 (2), 115-131.

Bull, P.y J. Elliot (1998). "Level of threat: Means of assessing interviewer toughness and neutrality". Journal of Language and Social Psychology, 17: 220-244.

Bull, P. y K. Mayer (1993). "How not to answer questions in political interviews". Political Psychology, 14, 651-66.

Byron, D. y P. A. Heeman (1997). "Discourse marker use in task-oriented spoken dialog”. Proceedings of Eurospeech'97. Internet <http://www.cs.rochester.edu/trs/ai-trs. html> (25-05-2010).

Carter, R. y McCarthy, M. (2002). "From conversation to corpus: A dual analysis of a broadcast political interview". Windows on the World. Media Discourse in English, A. Sánchez-Macarro (ed.), Valencia, Universitat de Valencia.

Clayman, S. E. (1988). "Displaying neutrality in television news interviews". Social Problems, 35, 474-492.

-, (1991). "News interview openings: Aspects of sequential organization". Broadcast Talk, P. Scannell (ed.), London, Sage, 48-75.

—, (1992). "Footing in the achievement of neutrality: the case of news-interview discourse". Talk at Work. Interaction in Institutional Settings, P. Drew y J. Heritage (eds.), Cambridge, Cambridge University Press, 163-198.

-, (1993). "Reformulating the question: A device for answering questions in news interviews and press conferences". Text, 13, 159-188.

—, (2001). "Answers and evasions". Language in Society, 30 (3), 403-442.

Соскстоғт, R. y S. Соскскоғт (1992). Persuading People. An Introduction to Rhetoric, London, Macmillan.

Duchenne, G. B. (1990). The mechanism of human facial expression or an electro-physiological analysis of the expression of the emotions (A. Cuthbertson, Traductor), Cambridge, Cambridge University Press. (Trabajo original publicado 1862).

Edwards, D. (1994). "Script formulations: A study of event descriptions in conversation”. Journal of Language and Social Psychology, 13 (3), 211-247 
-, (1995). "Two to tango: Script formulations, dispositions, and rhetorical symmetry in relationship troubles talk". Research on Language and Social Interaction, 28 (4), 319-350.

-, (1997). Discourse and Cognition, London, Sage.

-,(2000). "Extreme Case Formulations: Softeners, Investment, and Doing Nonliteral". Research on Language and Social Interaction, 23(4): 347-73.

-, (2003). "Analyzing Racial Discourse: The Discursive Psychology of Mind-World Relationships". Analyzing Race Talk. Multidisciplinary Perspectives on the Research Interview, H. Van Den Berg, M. Wetherell y H. Houtkoop (eds.), Cambridge, C.U.P, 31-48.

Ekman, P., W. V. Friesen y P. Ellsworth (1972). Emotion in the Human Face: Guidelines for Research and an Integration of Findings, Elmsford, New York, Pergamon Press Inc.

Ekman, P., W. V. Friesen and M. O'Sullivan (1988). "Smiles when lying". Journal of Personality and Social Psychology, 54: 414-420.

Greatbatch, D. (1986). "Aspects of topical organization in news interviews: The use of agenda-shifting procedures by IEs". Media, Culture and Society, 8, 441-455.

—, (1988). "A turn-taking system for British news interviews" en Language in Society, 17, 401-30.

—, (1992). "On the Management of Disagreement between News Interviewees" en P. Drew and J. Heritage (eds.) Talk at Work. Cambridge, England: Cambridge University Press, 268-301.

Hall, E. T. (1973). The Silent Language, New York, Anchor Press.

Harris, R. M. y D. Rubinstein (1975). "Paralanguage, Communication, and Cognition". Organization of Behavior in Face-to-Face Interaction, A. Kendon, R. M. Harris y M. R. Key (eds.), The Hague, Mouton Publishers, 251-276.

Harris, S. (1986). "IRs' questions in broadcast interviews". Belfast Working Papers in Language and Linguistics, J. Wilson y B. Crow (eds.), Jordanstown, University of U1ster, 50-85.

-, (1991). "Evasive actions: How politicians respond to questions in political interviews”. Broadcast Talk, P. Scannell (ed.), London, Sage, 76-99.

Hellweg, S., M. Pfau and S. R. Brydon (1992). Televised Presidential Debates: Advocacy in Contemporary America, New York, Praeger.

Heritage, J. y D. Greatbatch (1991). "On the institutional character of institutional talk: The case of news interviews". Talk and Social Structure, D. Boden y D. H. Zimmerman (eds.), Cambridge, Polity Press, 95-129.

Heritage, J. y A. L. Roth (1995). "Grammar and institution: Questions and Questioning in the Broadcast News Interview". Research on Language and Social Interaction, 28, 1-60.

Heritage, J. (2003). "Designing questions and setting agendas in the news interview". Studies in Language and Social Interaction, C. D. LeBaron y J. Mandelbaum (eds.), London, Lawrence Erlbaum, 57-90.

Knapp, M. L. (1980). Essentials of Nonverbal Communication, Chicago, Holt, Rinehart and Winston. 
McNeill, D. (1985). "So you think gestures are nonverbal?". Psychological Review, 92(3), 350-371.

Mehrabian, A. (1972). Nonverbal Communications, Aldine-Atherton, Chicago, Illinois.

Pomerantz, A. (1986). "Extreme Case Formulations: A Way of Legitimizing Claims". Human Studies, 9: 219-229.

Poyatos, F. (2002). Nonverbal Communication Across Disciplines, Amsterdam, John Benjamins.

Ramsey, S. J. (1979). "Nonverbal behavior: an intercultural perspective". Handbook of Intercultural Communication, M. K. Asante, E. Newmark y C. A. Blake (eds.), Beverly Hills, California, SAGE Publications, 105-143.

Richmond, V.P., J. C. McCroskey y S. K. Payne (1991). (Segunda edición) Nonverbal Behavior in Interpersonal Relations, Englewood Cliffs, New Jersey, Prentice-Hall, Inc. Schiffrin, D. (1985). "Conversational coherence: The role of well". Language, 61(3): 640-667.

-, (1987). Discourse Markers, Cambridge, New York, Cambridge University Press.

Sornig, K. (1989). “Some Remarks on Linguistic Strategies of persuasión”. Studies on Political Discourse, R. Wodak (ed.), Amsterdam, John Benjamins, 95-113

Tracey, M. (1977). The Production of Political Television, London, Routledge \& Kegan Paul.

Watzlawick, P., J. H. Beavin y D. D. Jackson (1967). Pragmatics of Human Communication: A study of Interactional Patterns, Pathologies, and Paradoxes, New York, W.W. Norton and Company, Inc.

Zillman, D. (1972). "Rhetorical Elicitation of Agreement in Persuasion". Journal of Personality and Social Psychology, 21:2, 159-165.

\section{AGRADECIMIENTOS}

Nos gustaría expresar nuestro agradecimiento a British Broadcasting Corporation por permitirnos reproducir en este artículo un gran número de citas de la entrevista Panorama Special. 